\title{
Special Issue: Predictive Multiscale Materials Modeling
}

\author{
M. A. Katsoulakis ${ }^{a}$ N. Zabaras ${ }^{b, c, d, *}$ \\ ${ }^{a}$ Center for Applied Mathematics and Computation and Department of Mathematics and \\ Statistics, University of Massachusetts Amherst, 710 N. Pleasant Street, Amherst, MA \\ 01003, USA \\ ${ }^{b}$ Department of Aerospace and Mechanical Engineering, University of Notre Dame, 372 \\ Fitzpatrick Hall, Notre Dame, IN 46556, USA \\ cInstitute for Advanced Study, Technische Universität München, Lichtenbergstr. 2 a, \\ D-85748 Garching, (b. München), Germany \\ ${ }^{d}$ Warwick Centre for Predictive Modeling, The University of Warwick, Coventry CV4 7AL, UK
}

Keywords: Materials Modeling, Uncertainty Quantification, Big Data, Predictive Modeling, Scientific Computing, Machine Learning, Coarse Graining

The motivation for this special issue stems from the symposium on "Predictive Multiscale Materials Modeling" that took place at the Isaac Newton Institute (INI) for Mathematical Sciences, University of Cambridge, during December 1-4, 2015. The symposium was hosted and and organized by the Turing Gateway to Mathematics (TGM) program at INI. Several leading scientists, from materials physics and chemistry, computational mathematics and statistics, machine learning, and scientific computing, engaged in discussions and exchanged ideas for four days with emphasis on predictability of multiscale materials simulations. This special issue contains a subset of the presentations. Video and slides of all the presentations are available on the INI-TGM website

\footnotetext{
*Corresponding Author.

Email addresses: markos@math . umass.edu (M. A. Katsoulakis ), nzabaras@gmail.com (N. Zabaras )

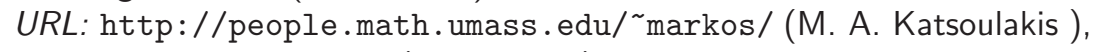
http:///www.zabaras.com/ (N. Zabaras )
}

Preprint submitted to Journal of Computational Physics

February 15, 2017

(C) 2017. This manuscript version is made available under the Elsevier user license http://www.elsevier.com/open-access/userlicense/1.0/ 
(http://www.turing-gateway.cam.ac.uk/uqm_dec2015.shtml).

The multiscale and multiphysics nature of materials gives rise to extremely challenging and unique mathematical and computational problems for developing predictive models that perform well. In addition, there is a huge gap at present between state-of-the-art mathematical and computational methods for materials modeling and design and the tools routinely used by both experimentalists and industry. The ability to predict the properties of a range of materials with confidence is paramount to a number of industrial sectors, including but not limited to aerospace, pharmaceutical, electronics, automotive and energy sectors. These sectors are amongst those that will benefit from mathematical innovations that lead to the improved development and accelerated deployment of new materials. For example, the timeliness and cost-effectiveness of the materials engineering process for new materials can be significantly improved by placing more focus on computational predictive modeling and probabilistically-based materials engineering.

Despite their sophistication and continued enhancement, computational materials models do not always perform as desired in the sense of being able to explain observation data under different process conditions. Discrepancies between observations and predictions may be generally explained by uncertainties inherent in the modeling process. For instance, this may include model uncertainties at each scale e.g. phenomenological constitutive equations or approximations and errors in electronic structure calculations, coupling of behaviors occurring across scales from the continuum to the quantum scale, lack of experimental data needed to account for information not present in the models, using surrogate models e.g. cluster expansions in ab-initio simulations, material-specific 
interatomic potentials in molecular simulations, etc., and many more.

Moreover, some of the current challenges and bottlenecks in predictive multiscale materials models are directly related with the lack of suitable computational and mathematical techniques to address unique challenges that arise in materials modeling. For example, such challenges include our inability to model high-dimensionality problems (e.g. considering random structures across scales), accounting for information loss when coarse-graining, modeling of rare events (e.g. failure of materials and devices such as batteries or fuel cells), lack of scalable uncertainty quantification techniques capable for capturing correlations within and across scales, and other.

With the revolution that has taken place over recent years in the data sciences along with the rapid advances in high performance computing, there is an increased ability to collect structure and property data at different resolutions and scales. These include simulation data from computationally demanding simulators, but also experimental data of structure and property (e.g. structure data using widely available imaging techniques). The exploration of data-driven modeling of material systems is needed to effectively integrate the available data with physical models towards predictive multiscale materials modeling and design.

The workshop brought together perspectives from leading scientists in computational materials science, uncertainty quantification and machine learning communities and industry leaders to address some of the aforementioned challenges. The focus of the workshop was to identify areas where attention was needed to develop improved approaches to data-driven predictive multiscale materials modeling and design. Over the course of four days, the participants met to discuss and exchange ideas. 
The key conclusions reached over the course of the workshop are summarized as follows.

- Mathematical and statistical developments for data-driven approaches to predictive materials modeling are of paramount importance for the accelerated design of materials and processes in the presence of inherent, structure, process, model and epistemic uncertainties.

- The identification and integration of synergistic methodologies from different research fields (including materials physics and chemistry, uncertainty quantification and machine learning) towards predictive materials modeling. Relationships between different communities can be multidirectional and synergistic.

- The concurrent revolutions in data science, high performance computing and statistical/machine learning bring to focus fundamental trade-offs in predictive computation between on one hand, complex models prevalent in mathematics, applied sciences and engineering, and statistical, data-driven models in data science and statistics communities on the other. The multiscale, multi-physics, high-dimensional nature of the challenges in materials design requires an integration of the two approaches, necessitating fundamental advancements in data assimilation methods.

- Predictive modeling may be used to guide the efforts of experimentalists to areas that may be in need of more focus. In other words, predictive models should be used to help identify the most informative experimental simulations needed to predict a quantity of interest. Conversely, although in the same spirit, experimental data should be incorporated into models to 
improve predictive capabilities. For example, data mining approaches may unveil unexplored materials that are likely to have the desired properties.

- The identification of bottlenecks in the materials design process, occurring across mathematics, the physical sciences and data science. There is a large gap between state-of-the-art mathematical methods and the analysis tools used by experimentalists and industry. The methods discussed in the workshop have significant potential to reduce the cost, time, and risk of material system validation for advanced materials, assessment of new design configurations at the component level, and for assessing risk and capability at the system level. Mathematical innovations lead to rapid materials development and deployment by identifying fundamental problems in materials modeling and design.

- Recommendations to develop initiatives that address uncertainty quantification and error analysis when developing predictive models. Moreover, it is recognized that quantifying model uncertainty is as an important step in multiscale modeling where the behaviors across different scales are coupled. Model selection issues arise prominently in obtaining reduced order or coarse-grained physical models [1].

- Machine learning and data mining approaches can be used to predict materials properties or to distill large amounts of complex data into a comprehensive form. In particular, surrogate models and optimized experiment design for regression problems [2], approximate inference for Bayesian inference, manifold learning for reduced order models were among those discussed [3]. Furthermore, approaches based on clustering, deep networks 
and probabilistic graphical models were also discussed. New approaches were proposed for understanding and quantifying model bias in machine learning and data-driven coarse-graining methods [4].

- There are a key number of modeling challenges to be addressed. Amongst those included devising methods and protocols for addressing rare events and extreme value statistics such as those giving rise to materials failure [5]. Other challenges included modeling across different but coupled time and length scales incorporating multiple physics, developing probabilistic hierarchical and multiscale models, scalable uncertainty quantification, effective coarse graining [6], relative entropy and variational inference methods suitable for correlated data, dynamics and time series [4, 7, 8], efficient Lattice Monte Carlo, parallel tempering, and multicanonical sampling in molecular simulations [9], and exploring high-dimensional structure relations.

- Multiscale materials modeling currently involves phenomenological constitute models that best match limited experimental observations under limited processes. What are the effective variables needed to interpret data? How are they coupled and involved during processing? These and related issues were discussed by many participants. Various approaches were presented to address these issues many with a statistical flavor (Bayesian model selection, sparse modeling, etc.) [10, 11].

We hope that this issue will become a valuable resource for documenting key aspects of the state-of-the-art in data-driven, predictive, computational modeling and will stimulate further inter-disciplinary advances in this direction. Lecture presentations from the workshop are available on the TGM website 
(http://www.turing-gateway.cam.ac.uk) in video or slide format.

\section{Acknowledgement}

The organizers would like to express their gratitude to the Isaac Newton Institute (INI) for Mathematical Sciences and the Turing Gateway to Mathematics (TGM) at the University of Cambridge, UK, for organizing, supporting and hosting the symposium on "Predictive Multiscale Materials Modeling" in December 2015. Furthermore, the organizers would like to thank all speakers, participants and authors who made this special issue possible. The organization of the meeting became possible through support of EPSRC to the Warwick Centre for Predictive Modelling at the University of Warwick, UK (Grant No. EP/L027682/1). N.Z. acknowledges additional support from the EPSRC, the Royal Society through a Wolfson Research Merit Award and the Technische Universität München, Institute for Advanced Study for support through a Hans Fisher Senior Fellowship, funded by the German Excellence Initiative and the European Union Seventh Framework Programme under Grant Agreement No. 291763. The preparation of this document was while N.Z. was supported by the Defense Advanced Research Projects Agency (DARPA) EQUiPS program under subcontract 4000149789 with the Oak Ridge National Laboratory (ORNL). The research of M.A.K. was partially supported by the DARPA EQUiPS program under the grant W911NF1520122, by the Office of Advanced Scientific Computing Research, U.S. Department of Energy (DOE), under Contract No. DE-SC0010723, and by the National Science Foundation (NSF) under the grant DMS-1515712. 


\section{References}

[1] L. Ellam, N. Zabaras, M. Girolami, A bayesian approach to multiscale inverse problems with on-the-fly scale determination, Journal of Computational Physics 326 (2016) 115 - 140. doi:http://dx.doi.org/10.1016/j.jcp.2016.08.031.

URL http://wWw.sciencedirect.com/science/article/pii/S0021999116303886

[2] Y. Shin, D. Xiu, On a near optimal sampling strategy for least squares polynomial regression, Journal of Computational Physics 326 (2016) 931 - 946. doi:http://dx.doi.org/10.1016/j.jcp.2016.09.032 URL//WWW.sciencedirect.com/science/article/pii/S0021999116304533

[3] W. Xing, V. Triantafyllidis, A. Shah, P. Nair, N. Zabaras, Manifold learning for the emulation of spatial fields from computational models, Journal of Computational Physics 326 (2016) 666 - 690. doi:http://dx.doi.org/10.1016/j.jcp.2016.07.040. URL http://wWw.sciencedirect.com/science/article/pii/S0021999116303722

[4] M. A. Katsoulakis, L. Rey-Bellet, J. Wang, Scalable information inequalities for uncertainty quantification, Journal of Computational Physics (2017) doi:http://dx.doi.org/10.1016/j.jcp.2017.02.020. URL http://www.sciencedirect.com/science/article/pii/S0021999117301134

[5] R. Lipton, P. Sinz, M. Stuebner, Uncertain loading and quantifying maximum energy concentration Journal of Computational Physics $325 \quad$ (2016) 38 - 52. 
doi:http://dx.doi.org/10.1016/j.jcp.2016.07.010.

URL http://wWw.sciencedirect.com/science/article/pii/S0021999116302893

[6] F. S. Cipcigan, V. P. Sokhan, J. Crain, G. J. Martyna,

Electronic coarse graining enhances the predictive power of molecular simulation allowing challeng

Journal of Computational Physics 326 (2016) 222 - 233.

doi:http://dx.doi.org/10.1016/j.jcp.2016.08.030.

URL http://wwW.sciencedirect.com/science/article/pii/S0021999116303874

[7] M. Opper, An estimator for the relative entropy rate of path measures for stochastic differential ec Journal of Computational Physics $330 \quad$ (2017) 127 - 133. doi:http://dx.doi.org/10.1016/j.jcp.2016.11.021.

URL//WWW.sciencedirect.com/science/article/pii/S0021999116306076

[8] K. Gourgoulias, M. A. Katsoulakis, L. Rey-Bellet, Information criteria for quantifying loss of reversibility in parallelized $\{\mathrm{KMC}\}$, Journal of Computational Physics 328 (2017) 438 - 454. doi:http://dx.doi.org/10.1016/j.jcp.2016.10.031.

URL//WWW.sciencedirect.com/science/article/pii/S0021999116305290

[9] Y. Xu, P. M. Rodger, Mbar-enhanced lattice monte carlo simulation of the effect of helices on men Journal of Computational Physics 333 (2017) 128 - 141. doi:http://dx.doi.org/10.1016/j.jcp.2016.12.016. URL http://wWw.sciencedirect.com/science/article/pii/S0021999116306696

[10] M. Aldegunde, N. Zabaras, Kristensen, Quantifying uncertainties in first-principles alloy thermodynamics using cluster expansions, Journal of Computational Physics $323 \quad$ (2016) 17 - 44. 
doi:http://dx.doi.org/10.1016/j.jcp.2016.07.016.

URL http://www.sciencedirect.com/science/article/pii/S0021999116302972

[11] M. Schöberl, N. Zabaras, P.-S. Koutsourelakis, Predictive coarse-graining, Journal of Computational Physics $333 \quad$ (2017) $49 \quad$ - 77. doi:http://dx.doi.org/10.1016/j.jcp.2016.10.073.

URL//WWW.sciencedirect.com/science/article/pii/S0021999116306763 